Ana Carolina Carro'

(ํ) https://orcid.org/0000-0001-8180-1544

Rodrigo Dias Nunes ${ }^{1,2}$

○ https://orcid.org/0000-0002-2261-8253

\section{Ideação suicida como fator associado à síndrome de Burnout em estudantes de Medicina}

\author{
Suicidal ideation as a factor associated with Burnout syndrome in medical students
}

DOI: $10.1590 / 0047-2085000000302$

\section{RESUMO}

Objetivo: Identificar a prevalência e fatores associados à síndrome de Burnout nos estudantes de Medicina. Métodos: Estudo transversal com estudantes de Medicina de uma instituição privada de ensino na região Sul do Brasil. Utilizou-se o Maslach Burnout Inventory - Student Survey para a identificação da síndrome e um instrumento para a coleta de informações sociodemográficas, hábitos, rotina e situação acadêmica. Realizou-se análise descritiva dos dados e avaliaram-se associações entre as informações obtidas a partir do instrumento de coleta com a presença da síndrome de Burnout, utilizando-se razões de prevalência por meio do teste qui-quadrado ou teste de Fisher. Associações com valor de $p$ $<0,25$ foram inseridas em um modelo multivariado, em formato step-forward, utilizando-se regressão de Poisson. Foram consideradas significativas associações com valor de $p<0,05$. Resultado: Quinhentos e vinte e dois $(88,2 \%)$ alunos participaram da pesquisa. A prevalência da síndrome de Burnout foi de 12,3\%. As associações com síndrome de Burnout que possuíram valor de $p<0,25$ foram "não viver com parceiro", "residir sozinho", "dormir até cinco horas por dia", "ter até cinco horas semanais de lazer", "possuir pensamento suicida" e "realizar acompanhamento psicológico". Após inserir essas variáveis no modelo multivariado, identificou-se apenas 10\% de maior probabilidade de síndrome de Burnout no estudante que relatou pensamento suicida, sendo esse o único fator associado encontrado à ocorrência de burnout $(p<0,001)$. Conclusão: A prevalência de síndrome de Burnout entre os acadêmicos de Medicina foi de 12,3\%, e apenas a presença de pensamentos suicidas durante o curso foi associada à sua ocorrência.

\section{PALAVRAS-CHAVE}

Burnout, ideação suicida, estudantes de Medicina.

\section{ABSTRACT}

Objective: To identify the prevalence and factors associated with Burnout syndrome in medical students. Methods: Cross-sectional study with medical students from a private educational institution in southern Brazil. The Maslach Burnout Inventory - Student Survey was used to identify the syndrome and an instrument for collecting sociodemographic information, habits, routine and academic situation. Descriptive analysis of the data was carried out and associations between related information were assessed using the collection instrument with the presence of Burnout syndrome, using the prevalence ratios using the chi-test or Fisher's test. Associations with a p-value $<0.25$ were inserted in a multivariate model, in a step-forward format, using Poisson regression. They were considered signed with p value < 0.05. Result: Five hundred and twenty-two (88.2\%) students participated in the survey. The prevalence of Burnout syndrome was $12.3 \%$. Associations with Burnout syndrome that had a value of $p<0.25$ were "not living with a partner", "living alone", "sleeping up to five hours a day", "having up to five hours a week of leisure", "having thoughts suicide" and "psychological counseling". After inserting these variables in the multivariate model, only $10 \%$ higher likelihood of Burnout syndrome was identified in the student who reported suicidal thinking, which is the only associated factor found with the occurrence of burnout $(p<0.001)$. Conclusion: The prevalence of Burnout syndrome among medical students was $12.3 \%$ and only the presence of suicidal thoughts during the course was associated with its occurrence.

\section{KEYWORDS}

Burnout, suicidal ideation, medical students.
Received in: July/6/2020. Approved in: Nov/2/2020

1 Universidade do Sul de Santa Catarina, Faculdade de Medicina, Palhoça, SC, Brasil.

2 Universidade do Sul de Santa Catarina, Programa de Pós-Graduação em Ciências da Saúde, Palhoça, SC, Brasil.

Address for correspondence: Ana Carolina Carro. Av. Trompowsky, 245, Centro - 88015-300 - Florianópolis, SC, Brasil. Telefone: +55 (48) $99164-1708$.

E-mail: anacarolinacarro@hotmail.com 


\section{INTRODUÇÃO}

A síndrome de Burnout, do inglês to burn out, traduzido como "esgotar-se", foi descrita pela primeira vez em 1974, pelo psicanalista Herbert Freudenberger, ao observar a diminuição do prazer que seu trabalho proporcionava. Dessa forma, a atividade laboral foi relacionada como agente causador de um estresse contínuo'. Em 1981, a síndrome de Burnout foi definida por Maslach e Jackson como uma síndrome tridimensional: exaustão emocional, despersonalização e falta de realização profissional². Tendo esse tripé como base, o indivíduo passa sofrer com suas consequências: humor deprimido, distanciamento afetivo, redução do prazer proporcionado pelas conquistas laborais, além de sintomas físicos como mialgia, cefaleia, distúrbios do sono, gastrointestinais e respiratórios, entre outros ${ }^{3}$.

Globalmente, o conhecimento acerca da prevalência da síndrome de Burnout é escasso 4 . Sabe-se que essa condição clínica vem se tornando cada vez mais prevalente, no entanto não há números específicos que abranjam a população mundial ${ }^{4}$. Isso ocorre pela ausência de critérios diagnósticos bem definidos e mundialmente aceitos ${ }^{4,5}$. Embora o instrumento mais comumente utilizado para mensurar a síndrome seja o Maslach Burnout Inventory (MBI), cada país possui uma forma de definir a existência de síndrome de Burnout em sua população, gerando discrepâncias entre os dados ${ }^{4,5}$.

Diferentemente da depressão, a síndrome de Burnout possui uma relação específica com o contexto da realidade vivenciada no ambiente do trabalho, enquanto a primeira abrange todas as áreas da vida de um indivíduo ${ }^{6}$. No entanto, as repercussões para quem desenvolve tal síndrome ultrapassam as esferas laborais e atingem o indivíduo em seus diferentes níveis: institucional, social e pessoa ${ }^{3,6}$.

Conhecendo a relação existente entre a síndrome de Burnout e o trabalho, realizaram-se estudos a fim de elucidar quais são as atividades profissionais mais acometidas. Notou-se alta prevalência de síndrome de Burnout em meios profissionais nos quais o nível de estresse do dia a dia era consideravelmente elevado 4 . Estão nesse grupo os profissionais da área da saúde, particularmente os médicos, com prevalência de síndrome de Burnout duas a três vezes maior do que em quaisquer outros profissionais ${ }^{7,8}$. Mundialmente, um a cada dois médicos desenvolve síndrome de Burnout. Desses, um terço é afetado consideravelmente e um décimo chega a formas graves em circunstâncias irrecuperáveis $5^{3,7}$. Porém, como já mencionado, a ausência de um padrão diagnóstico para a síndrome de Burnout dificulta a estimativa de prevalência de tal problema nessa população, deixando esse número variando de $0 \%$ a 80,5\% de síndrome de Burnout em médicos ${ }^{6}$.

A origem dessa relação está na realidade vivida por quem exerce a medicina, fator gerador do estresse emocional desencadeador da síndrome de Burnout ${ }^{8}$. Porém, muitas dessas reações já existem antes mesmo da vida profissional, podendo ser desencadeadas ainda durante a graduação, enquanto acadêmicos de Medicina9,10.

Aventou-se a hipótese de a síndrome de Burnout ser despertada precocemente, ainda nos anos preparatórios para o exercício da profissão, considerando-se que a tridimensionalidade poderia ser estabelecida pelo desgaste mental gerado pelo período de formação médica. Levando em conta tal hipótese, identificar possíveis características em comum entre portadores de síndrome de Burnout possibilita uma associação do perfil acadêmico de cada estudante com o desenvolvimento dessa síndrome. Além disso, a identificação precoce da síndrome de Burnout possibilita intervenções mais eficazes que impeçam o estudante de iniciar sua vida profissional com consequências herdadas dos seus anos preparatórios. Portanto, o objetivo deste estudo foi identificar a prevalência e os fatores associados à síndrome de Burnout em estudantes do curso de Medicina.

\section{MÉTODOS}

Foi realizado um estudo observacional de delineamento transversal envolvendo todos os alunos do curso de Medicina em uma grande universidade privada da região Sul do Brasil. O estudo ocorreu na Universidade do Sul de Santa Catarina, entre fevereiro de 2019 e junho de 2020, e foi realizado um censo entre os alunos do curso de Medicina dessa universidade, com estimativa de 625 estudantes. Incluíramse todos os alunos regularmente matriculados no curso de Medicina dessa universidade.

Após a abordagem dos estudantes pelo pesquisador e mediante a assinatura de um termo de consentimento livre e esclarecido, o instrumento foi aplicado em ambiente calmo e acolhedor. Para a coleta de dados, foi utilizado um questionário denominado Maslach Burnout Inventory - Student Survey (MBI-SS), visando à identificação da síndrome de Burnout. O MBI-SS foi desenvolvido por Schaufeli et al." para avaliar especificamente estudantes. Neste estudo, aplicou-se uma validação para estudantes brasileiros feita por Campos e Maroco ${ }^{12}$, composta por 15 itens com uma escala para respostas do tipo Likert, variando de zero (nunca) a seis (todos os dias), dividido em três dimensões: exaustão emocional (5 itens), despersonalização (4 itens) e realização profissional (6 itens). Feita a soma dos escores obtidos nos respectivos itens de cada dimensão, o ponto de corte para a determinação da exaustão emocional e despersonalização foi o percentil 66 (P66) e para a realização profissional, o percentil 33 (P33). Caracterizou-se como acometido pela síndrome de Burnout o indivíduo que apresentou simultaneamente valores acima do P66 dos escores para exaustão emocional e despersonalização e abaixo do P33 dos escores para realização profissional13. 
As informações sociodemográficas e sobre hábitos, rotina e situação acadêmica relacionadas ao perfil do estudante foram coletadas por meio de um instrumento desenvolvido para essa finalidade.

A análise dos dados ocorreu por meio do programa Statistical Package for the Social Sciences (SPSS), Version 18.0 [Computer program], Chicago: SPSS Inc; 2009. Na análise descritiva, os dados qualitativos foram apresentados na forma de frequência simples e relativa, já os dados quantitativos foram descritos em medidas de tendência central (média ou mediana) e suas respectivas medidas de dispersão. Avaliaram-se as associações entre as características sociodemográficas, os hábitos, a rotina e a situação acadêmica do estudante com a presença da síndrome de Burnout, utilizando-se as razões de prevalência (RPs) e seus respectivos intervalos de confiança de $95 \%$ (IC95\%), por meio do teste qui-quadrado ou prova exata de Fisher. As associações com valor de $p<0,25$ foram, posteriormente, inseridas em um modelo multivariado, em formato step-forward, utilizando-se a regressão de Poisson, aplicado para o ajuste do efeito independente da exposição no desfecho de interesse. Foram consideradas significativas as associações com valor de $p<0,05$.

Os riscos do estudo eram mínimos e consistiam em um possível desconforto com algumas perguntas contidas no instrumento de coleta de dados. Para minimizá-los, salientou-se, na entrega do questionário, que o(a) participante possuía total liberdade para não responder aos questionamentos ou interromper o preenchimento em qualquer momento, caso houvesse alguém desconforto. Foi reforçada a ideia de que o anonimato dos dados na coleta e na publicação dos resultados seria garantido.

Um Termo de Consentimento Livre e Esclarecido foi impresso em duas vias, sendo uma para ser entregue ao estudante e outra para ser assinada por ele e devolvida ao pesquisador antes do preenchimento do questionário. O estudo foi aprovado pelo Comitê de Ética em Pesquisa da Universidade do Sul de Santa Catarina com o CAAE 11168919.3.0000.5369.

\section{RESULTADOS}

Todos os alunos matriculados no curso de Medicina da Universidade do Sul de Santa Catarina foram convidados a participar da pesquisa. Desses, 522 (88,2\%) aceitaram, respondendo ao instrumento de pesquisa proposto. A idade dos entrevistados variou de 17 a 37 anos, com média de 22,2 $\pm 3,4$ anos. A prevalência da síndrome de Burnout na população estudada foi de $12,3 \%$. Foram realizadas análises de associações entre as características sociodemográicas (Tabela 1), hábitos e rotina (Tabela 2) e situação acadêmica (Tabela 3) do estudante com a existência da síndrome de Burnout.
Na análise bivariada, as características que possuíram valor de $p<0,25$, quando relacionadas com síndrome de Burnout, foram "não viver com parceiro" $(p=0,145)$, "residir sozinho" ( $p=0,245)$, "dormir até cinco horas por dia" $(p=0,120)$, "ter até cinco horas semanais de lazer" $(p=0,205)$, "possuir pensamento suicida" ( $p<0,001)$ e "realizar acompanhamento psicológico" $(p=0,028)$.

As variáveis citadas anteriormente foram inseridas em um modelo multivariado em formato step-forward. Em um primeiro bloco, foram analisadas as variáveis sociodemográficas "não viver com parceiro" e "residir sozinho". Posteriormente, a variável "não viver com um parceiro" $(p=0,021)$ foi inserida no segundo bloco de variáveis relacionadas aos hábitos e rotina do estudante "dormir até cinco horas por dia" e "ter até cinco horas semanais de lazer". As três variáveis demonstraram valores de $p<0,25$. Todas foram inseridas no terceiro e último bloco da situação acadêmica com "possuir pensamento suicida" e "realizar acompanhamento psicológico" (Tabela 4).

O resultado identificou não haver associação da síndrome de Burnout com as características sociodemográficas, os hábitos e a rotina do aluno e praticamente todas as variáveis relacionadas à situação acadêmica do aluno do curso de Medicina. Porém, o aluno que relatou pensamento suicida possuiu $10 \%$ de maior probabilidade de apresentar concomitantemente a síndrome de Burnout. Dessa forma, a única variável que demonstrou ter associação com burnout foi "possuir pensamento suicida" durante o curso $(p<0,001)$.

\section{DISCUSSÃO}

Este estudo foi realizado com alunos da primeira à décima segunda fase do curso de Medicina por meio de um instrumento validado para medir o nível de síndrome de Burnout e outro elaborado pelos autores e que englobou questões sociodemográficas e de hábitos, rotina e situação acadêmica do estudante. A considerável taxa de resposta obtida $(88,2 \%)$ pode ser decorrente da conscientização a respeito da importância do tema pesquisado e do envolvimento estudantil como sujeitos de pesquisa, assim possibilitando uma análise dos resultados com o controle de possíveis variáveis confundidoras.

Foi identificada uma prevalência de $12,3 \%$ de síndrome de Burnout nos estudantes de Medicina, usando como critério a necessidade de ser positivo nas três dimensões do MBISS. Utilizando-se os mesmos parâmetros, foram encontradas, em Barbosa et al. ${ }^{14}$, a taxa de 12\%, entre 399 alunos, e, em Almeida et al. ${ }^{15}$, a de 14,9\%, entre 376 alunos. Ambos os estudos foram realizados em universidades brasileiras e utilizando do mesmo instrumento de pesquisa validado (MBI-SS), corroborando o resultado encontrado. 
Tabela 1. Análise bivariada comparativa entre as características sociodemográficas do estudante de Medicina e síndrome de Burnout - Palhoça, SC, Brasil, $2019(N=522)$

\begin{tabular}{|c|c|c|c|c|}
\hline \multirow{3}{*}{ Características sociodemográficas } & \multicolumn{3}{|c|}{ Sindrome de Burnout } & \multirow{3}{*}{ Valor de $p$} \\
\hline & Sim & Não & Total & \\
\hline & n (\%) & $n(\%)$ & $n(\%)$ & \\
\hline \multicolumn{5}{|l|}{ Idade } \\
\hline$\leq 21$ anos & $28(11,1)$ & $225(88,9)$ & $253(48,5)$ & 0,420 \\
\hline$>21$ anos & $36(13,4)$ & $233(86,6)$ & $269(51,5)$ & \\
\hline \multicolumn{5}{|l|}{ Sexo } \\
\hline Masculino & $21(11,2)$ & $166(88,8)$ & $187(35,8)$ & 0,592 \\
\hline Feminino & $43(12,8)$ & $292(87,2)$ & $335(64,2)$ & \\
\hline \multicolumn{5}{|l|}{ Cor de pele } \\
\hline Branca & $62(12,1)$ & $452(87,9)$ & $514(98,5)$ & 0,256 \\
\hline Não branca & $2(25,0)$ & $6(75,0)$ & $8(1,5)$ & \\
\hline \multicolumn{5}{|l|}{ Possui parceiro } \\
\hline $\operatorname{Sim}$ & $2(4,7)$ & $41(95,3)$ & $43(8,2)$ & 0,145 \\
\hline Não & $62(12,9)$ & $417(87,1)$ & $479(91,8)$ & \\
\hline \multicolumn{5}{|l|}{ Filhos } \\
\hline $\operatorname{Sim}$ & $0(0,0)$ & $9(100,0)$ & $9(1,7)$ & 0,609 \\
\hline Não & $64(12,5)$ & $449(87,5)$ & $513(98,3)$ & \\
\hline \multicolumn{5}{|l|}{ Atividade laboral } \\
\hline $\operatorname{Sim}$ & $2(10,0)$ & $18(90,0)$ & $20(3,8)$ & 1,000 \\
\hline Não & $62(12,4)$ & $440(87,6)$ & $502(996,2)$ & \\
\hline \multicolumn{5}{|l|}{ Meio de sustento } \\
\hline Renda própria & $3(9,7)$ & $28(90,3)$ & $31(5,9)$ & 1,000 \\
\hline Renda familiar & $61(12,4)$ & $430(87,6)$ & $491(94,1)$ & \\
\hline \multicolumn{5}{|l|}{ Renda mensal } \\
\hline <4 salários mínimos & $12(13,6)$ & $76(86,4)$ & $88(16,9)$ & 0,666 \\
\hline \4 salários mínimos & $52(12,0)$ & $382(88,0)$ & $434(83,1)$ & \\
\hline \multicolumn{5}{|l|}{ Reside com } \\
\hline Sozinho & $29(10,7)$ & $243(89,3)$ & $272(52,1)$ & 0,245 \\
\hline Família & $35(14,0)$ & $215(86,0)$ & $250(47,9)$ & \\
\hline \multicolumn{5}{|l|}{ Problema de saúde } \\
\hline Não & $41(11,7)$ & $309(88,3)$ & $350(67,0)$ & 0,587 \\
\hline $\operatorname{Sim}$ & $23(13,4)$ & $149(86,6)$ & $172(33,0)$ & \\
\hline \multicolumn{5}{|l|}{ Uso regular de medicação } \\
\hline Não & $37(12,0)$ & $272(88,0)$ & $309(59,2)$ & 0,810 \\
\hline Sim & $27(12,7)$ & $186(87,3)$ & $213(40,8)$ & \\
\hline
\end{tabular}

Tabela 2. Análise bivariada comparativa entre as características de hábitos e rotina do estudante de Medicina e síndrome de Burnout - Palhoça, SC, Brasil, 2019 ( $N=522)$

\begin{tabular}{|c|c|c|c|c|}
\hline \multirow[b]{2}{*}{ Características de hábitos e rotina } & \multicolumn{3}{|c|}{ Síndrome de Burnout } & \multirow[b]{2}{*}{ Valor de $\mathrm{p}$} \\
\hline & $\begin{array}{c}\text { Sim } \\
\text { n (\%) }\end{array}$ & $\begin{array}{c}\text { Não } \\
\text { n (\%) }\end{array}$ & $\begin{array}{l}\text { Total } \\
\text { n (\%) }\end{array}$ & \\
\hline \multicolumn{5}{|l|}{ Horas diárias de sono } \\
\hline$\leq 5$ horas & $56(11,6)$ & $426(88,4)$ & $482(92,3)$ & 0,120 \\
\hline$>5$ horas & $8(20)$, & $32(80,0)$ & $40(7,7)$ & \\
\hline \multicolumn{5}{|l|}{ Atividade física } \\
\hline Sim & $36(12,8)$ & $246(87,2)$ & $282(54,0)$ & 0,703 \\
\hline Não & $21(11,7)$ & $212(88,3)$ & $240(46,0)$ & \\
\hline \multicolumn{5}{|l|}{ Horas semanais de lazer } \\
\hline$\leq 5$ horas & $23(10,2)$ & $203(89,8)$ & $226(43,3)$ & 0,205 \\
\hline$>5$ horas & $41(13,9)$ & $255(86,1)$ & $296(56,7)$ & \\
\hline \multicolumn{5}{|l|}{ Frequência na presença da família } \\
\hline$\leq 1$ vez na semana & $25(10,7)$ & $208(89,3)$ & $233(44,6)$ & 0,338 \\
\hline$>2$ vezes na semana & $39(13,5)$ & $250(86,5)$ & $289(55,4)$ & \\
\hline
\end{tabular}


Tabela 3. Análise bivariada comparativa entre as características da situação acadêmica do estudante de Medicina e síndrome de Burnout - Palhoça, SC, Brasil, 2019 ( $N$ = 522)

\begin{tabular}{|c|c|c|c|c|}
\hline \multirow{3}{*}{ Características da situação acadêmica } & \multicolumn{3}{|c|}{ Síndrome de Burnout } & \multirow{3}{*}{ Valor de } \\
\hline & Sim & Não & Total & \\
\hline & n (\%) & n (\%) & n (\%) & \\
\hline \multicolumn{5}{|l|}{ Fase $(1-4 / 5-8)$} \\
\hline 1 a 4 & $27(10,6)$ & $227(89,4)$ & $254(64,1)$ & 0,224 \\
\hline 5 a 8 & $21(14,8)$ & $121(85,2)$ & $142(35,9)$ & \\
\hline \multicolumn{5}{|l|}{ Fase (1-4/9-12) } \\
\hline 1 a 4 & $27(10,6)$ & $227(89,4)$ & $254(66,8)$ & 0,549 \\
\hline 9 a 12 & $16(12,7)$ & $110(87,3)$ & $126(33,2)$ & \\
\hline \multicolumn{5}{|l|}{ Mudança de residência para cursar a faculdade } \\
\hline Não & $32(12,1)$ & $233(87,9)$ & $265(50,8)$ & 0,896 \\
\hline Sim & $32(12,5)$ & $225(87,5)$ & $257(49,2)$ & \\
\hline \multicolumn{5}{|l|}{ Bolsa ou financiamento } \\
\hline Não & $40(11,2)$ & $316(88,8)$ & $356(68,2)$ & 0,296 \\
\hline Sim & $24(14,5)$ & $142(85,5)$ & $166(31,8)$ & \\
\hline \multicolumn{5}{|l|}{ Modo de custeio dos estudos } \\
\hline Família ou si próprio & $41(11,7)$ & $309(88,3)$ & $350(67,0)$ & 0,587 \\
\hline Financiamento/bolsa & $23(13,4)$ & $149(86,6)$ & $172(33,0)$ & \\
\hline \multicolumn{5}{|l|}{ Horas semanais de estudo } \\
\hline$\leq 30$ horas & $15(12,1)$ & $109(87,9)$ & $124(23,8)$ & 0,949 \\
\hline$>30$ horas & $49(12,3)$ & $349(87,7)$ & $398(76,2)$ & \\
\hline \multicolumn{5}{|l|}{ Atividade extracurricular do curso } \\
\hline Não & $31(12,9)$ & $209(87,1)$ & $240(46,0)$ & 0,673 \\
\hline Sim & $33(11,7)$ & $249(88,3)$ & $282(54,0)$ & \\
\hline \multicolumn{5}{|l|}{ Horas de atividade extracurricular } \\
\hline$<3$ horas & $40(13,0)$ & $268(87,0)$ & $308(59,0)$ & 0,544 \\
\hline$\geq 3$ horas & $24(11,2)$ & $190(88,8)$ & $214(41,0)$ & \\
\hline \multicolumn{5}{|l|}{ Satisfação com o curso } \\
\hline Não & $5(9,1)$ & $50(90,9)$ & $55(10,5)$ & 0,663 \\
\hline Sim & $59(12,6)$ & $408(87,4)$ & $467(89,5)$ & \\
\hline \multicolumn{5}{|l|}{ Pensar em desistir do curso } \\
\hline Não & $46(11,9)$ & $340(88,1)$ & $386(73,9)$ & 0,687 \\
\hline Sim & $18(13,2)$ & $118(86,8)$ & $136(26,1)$ & \\
\hline \multicolumn{5}{|l|}{ Uso de medicação para estudar } \\
\hline Não & $40(11,4)$ & $310(88,6)$ & $350(67,0)$ & 0,408 \\
\hline Sim & $24(14,0)$ & $148(86,0)$ & $172(33,0)$ & \\
\hline \multicolumn{5}{|l|}{ Pensamentos suicidas durante 0 curso } \\
\hline $\operatorname{Sim}$ & $37(8,7)$ & $387(91,3)$ & $424(81,2)$ & $<0,001$ \\
\hline Não & $27(27,6)$ & $71(72,4)$ & $98(18,8)$ & \\
\hline \multicolumn{5}{|c|}{ Acompanhamento psicológico devido aos estudos } \\
\hline Sim & $36(10,1)$ & $320(89,9)$ & $356(68,2)$ & 0,028 \\
\hline Não & $28(16,9)$ & $138(83,1)$ & $166(31,8)$ & \\
\hline
\end{tabular}

Assim como em Almeida et al. ${ }^{15}$, não foi encontrada nenhuma associação de síndrome de Burnout com variáveis sociodemográficas. Em paralelo, uma recente metanálise feita por Frajerman et al. ${ }^{9}$ evidenciou a prevalência de síndrome de Burnout variando de acordo com o continente em que o estudante vivia. Isso nos mostra que, entre as características que marcam a população brasileira e a homogeneidade das qualidades que norteiam a maioria dos estudantes de Medicina do nosso país (brancos, solteiros, sem filhos e sem necessidade de sustento próprio), pouca associação se encontra com o desenvolvimento de síndrome de Burnout. Notamos o reflexo que isso pode gerar no acometimento dos alunos pela síndrome de Burnout e a influência que isso pode ter na qualidade de saúde mental de um estudante de Medicina, bem como de outros indivíduos daquela população, quando extrapolamos essa visão para uma esfera mundial, onde a realidade desses acadêmicos varia de acordo com o local em que vivem e onde estão expostos a conflitos internos, discriminação de gênero, dificuldades sociais, entre outros. 
Tabela 4. Análise multivariada entre as características dos estudantes de Medicina e a síndrome de Burnout - Palhoça, SC, Brasil, 2019 (N = 522)

\begin{tabular}{|c|c|c|c|c|}
\hline \multirow{2}{*}{ Características } & \multicolumn{4}{|c|}{ Síndrome de Burnout } \\
\hline & $\mathrm{RP}_{\mathrm{b}}(\mathrm{IC} 95 \%)$ & ${ }^{*}$ Valor de p & $\mathrm{RP}_{\mathrm{a}}$ (IC95\%) & ${ }^{\star \star}$ Valor de $\mathrm{p}$ \\
\hline \multicolumn{5}{|l|}{ Vive com parceiro } \\
\hline $\operatorname{Sim}$ & $0,18(0,01-0,08)$ & 0,021 & $1,04(1,00-1,08)$ & 0,390 \\
\hline Não & 1,00 & & 1,00 & \\
\hline \multicolumn{5}{|l|}{ Reside com } \\
\hline Sozinho & $0,15(0,05-0,01)$ & 0,270 & $\#$ & \\
\hline Família & 1,00 & & & \\
\hline \multicolumn{5}{|c|}{ Horas diárias de sono } \\
\hline$\leq 5$ horas & $0,36(0,03-0,11)$ & 0,250 & $1,03(0,96-1,11)$ & 0,371 \\
\hline$>5$ horas & 1,00 & & 1,00 & \\
\hline \multicolumn{5}{|c|}{ Horas semanais de lazer } \\
\hline$\leq 5$ horas & $0,15(0,01-0,05)$ & 0,213 & $1,01(0,99-1,04)$ & 0,333 \\
\hline$>5$ horas & 1,00 & & 1,00 & \\
\hline \multicolumn{5}{|c|}{ Pensamentos suicidas durante 0 curso } \\
\hline Sim & $0,03(0,04-0,15)$ & 0,001 & $1,10(1,04-1,16)$ & 0,001 \\
\hline Não & 1,00 & & 1,00 & \\
\hline \multicolumn{5}{|c|}{ Acompanhamento psicológico devido aos estudos } \\
\hline $\operatorname{Sim}$ & $0,02(0,06-0,02)$ & 0,269 & $0,98(0,94-1,02)$ & 0,269 \\
\hline Não & 1,00 & & 1,00 & \\
\hline
\end{tabular}

$\mathrm{RP}_{\mathrm{b}}$ : razão de prevalência bruta; $\mathrm{RP}_{\mathrm{a}}$ : razão de prevalência ajustada por regressão de Poisson.

*Valor de $\mathrm{p}$ bruto. * Valor de $\mathrm{p}$ ajustado pelas outras variáveis do modelo.

A prevalência de síndrome de Burnout encontrada no atual estudo e a falta de associação com hábitos de vida e quase todas as variáveis relacionadas à situação acadêmica do estudante não necessariamente refletem a ausência de prejuízos da saúde mental. Em um estudo publicado no JAMA em 2016, observou-se uma mediana no aumento dos sintomas depressivos de 13,5\%, comparando antes e após a faculdade de Medicina ${ }^{16}$. Em outro estudo, observou-se que a autopercepção do estudante de Medicina em relação ao prejuízo da sua saúde mental é de $36 \%$ antes da entrada no curso, enquanto $47 \%$ notam uma piora ao longo dos anos de vida acadêmica ${ }^{17}$. Na população estudada, ao responderem sobre a necessidade de iniciarem algum tipo de acompanhamento psicológico ou psiquiátrico por causa dos estudos, 68,2\% afirmaram ter tal necessidade, sendo esse acompanhamento feito com psicólogo, psiquiatra ou ambos. Esse dado possui certa ambivalência, podendo ser visto por um lado positivo, que mostra o baixo estigma da necessidade de acompanhamento psiquiátrico ou psicológico, e por um lado negativo, representado pela alta demanda na procura por esses serviços.

Além disso, os participantes deste estudo relataram possuir diversas afecções relacionadas à saúde mental. Entre as mais citadas, estavam ansiedade (12,8\%), depressão $(11,1 \%)$ e déficit de atenção (6,1\%). Em sua metanálise, Pacheco et al. ${ }^{18}$ observaram uma prevalência de $89,6 \%$ de sintomas ansiosos e $30,6 \%$ de quadros depressivos entre os acadêmicos do curso de Medicina. Ademais, segundo a metanálise realizada pelo JAMA ${ }^{16}, 27,2 \%$ dos estudantes apresentam depressão ou sintomas depressivos. Essa diferença de valores pode ser explicada pela não utilização de um instrumento validado para a aferição de diagnósticos de transtornos ansiosos e depressivos, como DASS-21, HAM-D e HAM-A. Em vez disso, utilizou-se o relato da autopercepção a respeito de algum problema de saúde. Dessa forma, a falta de conhecimento acerca desses problemas pode subestimar os valores identificados, devendo ser avaliados com cautela.

De forma significativa, estatisticamente, foi revelada uma associação entre ideação suicida e síndrome de Burnout. Essa relação foi observada pela primeira vez em um estudo com acadêmicos de Medicina dos Estados Unidos ${ }^{19}$. No entanto, desde então, não há muitas pesquisas que evidenciem esse fato, embora a ideação suicida sempre esteja presente em estudos referentes à síndrome de Burnout. Entre os alunos entrevistados, 81,2\% apresentaram algum tipo de pensamento suicida durante o curso. Esse valor é consideravelmente mais alto que outras taxas já encontradas, como em Torres et al. ${ }^{20}(7,2 \%)$ e Rotenstein et al. ${ }^{16}(11,1 \%)$. Outra revisão sistemática ${ }^{21}$ identificou um amplo intervalo na prevalência $(1,8 \%$ a $53,6 \%)$ de pessoas que compõem esse grupo. Isso ocorre por causa dos diferentes instrumentos utilizados para analisar a presença de ideação suicida, além do fato da diversidade da população estudada ao redor do mundo. Como nosso estudo não utilizou nenhum instrumento validado para analisar ideação suicida, como a Escala de Avaliação do Risco de Suicídio de Columbia, e apenas consideramos o relato pessoal de cada participante, isso explicaria o número aumentado de pessoas autorreferindo tal ideação. 
No entanto, a não utilização de um instrumento validado para o risco de suicídio não deve subestimar o que representa o dado encontrado neste estudo, visto que o relato por si só de pensamentos suicidas já deve ligar o alerta para o risco desse problema. Dessa forma, sendo essa a única variável estatisticamente significativa com o desfecho (síndrome de Burnout), aventa-se como hipótese que o desenvolvimento de síndrome de Burnout não esteja relacionado com a formação médica, mas que já venha na bagagem do estudante desde antes da faculdade.

A formação médica se inicia antes mesmo do curso de Medicina. O processo pelo qual o estudante passa para conquistar uma vaga na universidade é árduo e desgastante, exigindo do candidato sacrifícios que suplantam os de quem opta por qualquer outro curso. Já nessa etapa se iniciam as rotinas intensas de estudo, seguidas da abdicação de momentos com a família e com os amigos. Grace ${ }^{22}$ mostra em sua pesquisa o acometimento de estudantes norte-americanos pela depressão e síndrome de Burnout já na fase anterior à faculdade de Medicina. Ainda, relaciona esses prejuízos à saúde mental com a diminuição pelo interesse no curso. Assim, muitos dos estudantes, quando não desistem por causa dos inúmeros obstáculos, ingressam na Medicina trazendo consigo uma bagagem de danos à sua saúde mental. Somado a isso, a faculdade de Medicina possui características únicas que vão além dos demais cursos de ensino superior.

Os paradoxos vividos durante os anos de formação médica são constantes. A expectativa, a ansiedade, o receio e a falta de confiança inerentes a qualquer um que esteja começando seu processo de aprendizagem exemplificam essa dicotomia. Junto, tem-se a elevada carga horária característica do curso, que reserva pouco tempo para práticas de atividade física, lazer, boa alimentação e sono. Então, nota-se um acadêmico que chegou à faculdade com prejuízos à sua saúde mental sem a devida exploração e cuidados. Somam-se todos os percalços vividos pela rotina acadêmica, culminando em alguém que manifesta síndrome de Burnout durante o curso, porém não exclusivamente por causa dele, mas sim como o histórico inerente a todo o processo para alcançar seu objetivo. A falta de atenção e cuidados, além de estigmas que envolvem a depressão e a ansiedade, desenvolvidos e/ou agravados pela faculdade e manifestados por meio da síndrome de Burnout, levam o estudante a extremos como a ideação suicida. Assim, é possível entender a forte associação encontrada neste estudo entre síndrome de Burnout e ideação suicida.

Por se tratar de um estudo transversal, existem limitações que envolvem a impossibilidade de estabelecer relação causal entre as variáveis. Talvez o acompanhamento desses alunos, previamente à sua entrada na universidade e mantido durante o curso, pudesse contribuir para essa elucidação. Outro fator limitante importante foi a extensão do questionário elaborado pelos autores, que impossibilitou o uso de instrumentos validados para analisar as principais variáveis exploradas neste estudo, como ansiedade, depressão e ideação suicida. Além disso, esta amostra, apesar de significativa, representa apenas a realidade da população de um único curso de Medicina do Brasil.

\section{CONCLUSÃO}

A prevalência de síndrome de Burnout entre os acadêmicos de Medicina foi de 12,3\%, e apenas a presença de pensamentos suicidas durante o curso foi associada à ocorrência de burnout. Ademais, a falta de associações significativas com características acadêmicas infere a preexistência de dificuldades emocionais. Ainda, a alta prevalência de ideação suicida e outros achados, como o início da necessidade de acompanhamento psicológico após a entrada na universidade, não descartam a importância de se acompanhar de perto a saúde mental dessa população. Isso posto, é de extrema importância a realização de mais estudos na área da saúde mental não somente com estudantes de Medicina e médicos, mas também com aqueles que almejam essa formação.

\section{CONTRIBUIÇÕES INDIVIDUAIS}

Ana Carolina Carro - Contribuiu na concepção e desenho do estudo, coleta de dados, interpretação dos resultados, revisão de literatura e elaboração do manuscrito.

Rodrigo Dias Nunes - Foi orientador do estudo, realizou a análise estatística e contribuiu em todas as outras etapas de realização do estudo, incluindo a concepção e desenho do estudo, interpretação dos resultados, revisão de literatura, elaboração do manuscrito e revisão e aprovação final a ser publicada.

\section{CONFLITO DE INTERESSES}

Não há conflito de interesses. Não há fontes externas de subsídio para esta pesquisa.

\section{AGRADECIMENTOS}

Agradecemos ao curso de Medicina da UNISUL - Pedra Branca, pelo apoio dado para a realização deste trabalho, e aos seus respectivos alunos, pela participação nesta pesquisa.

\section{REFERÊNCIAS}

1. Freudenberger HJ. Staff burn-out. J Soc Issues. 1974;30(1):159-65. 
2. Maslach C, Jackson SE. The measurement of experienced burnout. J Organiz Behav. 1981;2(2):99113.

3. Salvagioni DAJ, Melanda FN, Mesas AE, González AD, Gabani FL, Andrade SM. Physical, psychological and occupational consequences of job burnout: A systematic review of prospective studies. PLoS One. 2017;12(10):e0185781.

4. Lastovkova A, Carder M, Rasmussen HM, Sjoberg L, Groene GJ, Sauni R, et al. Burnout syndrome as an occupational disease in the European Union: an exploratory study. Ind Health. 2018;56(2):160-5.

5. Rotenstein LS, Torre M, Ramos MA, Rosales RC, Guille C, Sen S. Prevalence of Burnout Among Physicians A Systematic Review. JAMA. 2018;320(11):1131-50.

6. Bianchi R, Schonfeld IS, Laurent E. Physician burnout is better conceptualised as depression. Lancet. 2017;389(1):1397-8.

7. Ziad K, Laurent B, Marianne H, Virginie V, Christophe L, Guillaume F. Burnout in French physicians: A systematic review and meta-analysis. J Affect Disord. 2018;246(1):132-47.

8. Gorgulu 0, Akilli A. The determination of the levels of burnout syndrome, organizational commitment, and job satisfaction of the health workers. Niger J Clin Pract. 2017;20(1):48-56.

9. Frajerman A, Morvan Y, Krebs MO, Gorwood P, Chaumette B. Burnout in medical students before residency: A systematic review and meta-analysis. Eur Psychiatry. 2019;55(1):36-42.

10. Rodrigues H, Cobucci R, Oliveira A, Cabral JV, Medeiros L, Gurgel K, et al. Burnout syndrome among medical residents: A systematic review and meta-analysis. PLoS One. 2018;13(11):1-17.

11. Schaufeli WB, Martínez IM, Pinto AM, Salanova M, Bakker AB. Burnout and Engagement in University Students: A Cross-National Study. J Cross Cult Psychol. 2002;33(5):464-81.

12. Campos JADB, Maroco J. Maslach Burnout Inventory - Student Survey: Portugal-Brazil crosscultural adaptation. Rev Saúde Pública. 2012;46(5):816-24.
13. Maroco J, Tecedeiro M. Inventário de Burnout de Maslach para estudantes portugueses. Psicol Saúde Doenças. 2009;10(2):227-35.

14. Barbosa ML, Ferreira BLR, Vargas TN, da Silva GMN, Nardi AE, Machado S, et al. Burnout Prevalence and Associated Factors Among Brazilian Medical Students. Clin Pract Epidemiol Ment Health. 2018;14(1):188-95.

15. Almeida GC, de Souza HR, de Almeida PC, Almeida BC, Almeida GH. The prevalence of burnout syndrome in medical students. Arch Clin Psychiatry. 2016;43(1):6-10.

16. Rotenstein LS, Ramos MA, Torre M, Segal JB, Peluso MJ, Guille C, et al. Prevalence of Depression, Depressive Symptoms, and Suicidal Ideation Among Medical Students A Systematic Review and Meta-Analysis. JAMA. 2016;316(21):2214-36.

17. Fletcher I, Castle M, Scarpa A, Myers O, Lawrence E. An exploration of medical student attitudes towards disclosure of mental illness. Med Educ Online. 2020;25(1):1-9.

18. Pacheco JP, Giacomin HT, Tam WW, Ribeiro TB, Arab C, Bezerra IM, et al. Mental health problems among medical students in Brazil: a systematic review and meta-analysis. Braz I Psychiatry. 2017;39(4):369-78.

19. Dyrbye LN, Thomas MR, Massie FS, Power DV, Eacker A, Harper W, et al. Burnout and Suicidal Ideation among U.S. Medical Students. Ann Intern Med. 2008;149(5):334-41.

20. Torres AR, Campos LM, Lima MCP, Cerqueira ATAR. Suicidal Ideation Among Medical Students. J Nerv Ment Dis. 2018;206(3):160-8.

21. Coentre R, Góis C. Suicidal ideation in medical students: recent insights. Adv Med Educ Pract. 2018;9(1):873-80

22. Grace MK. Depressive symptoms, burnout, and declining medical career interest among undergraduate premedical students. Int J Med Educ. 2018;9(1):302-8. 